\title{
A uniqueness result for a singular elliptic equation with gradient term
}

\section{José Carmona}

Departamento de Matemáticas, Universidad de Almería, Carretera Sacramento S/N, La Cañada de San Urbano, 04120 Almería, Spain (jcarmona@ual.es)

\section{Tommaso Leonori*}

Departamento de Análisis Matemático, Campus Fuentenueva S/N, Universidad de Granada, 18071 Granada, Spain (leonori@ugr.es)

(MS received 1 July 2016; accepted 13 November 2016)

We prove the uniqueness of a solution for a problem whose simplest model is

$$
\left.\begin{array}{rlrl}
-\Delta u+\frac{k}{u}|D u|^{2} & =f(x) & & \text { in } \Omega, \\
u & =0 & & \text { on } \partial \Omega,
\end{array}\right\}
$$

with $k \geqslant 1,0 \lesseqgtr f \in L^{\infty}(\Omega)$ and $\Omega$ is a bounded domain of $\mathbb{R}^{N}, N \geqslant 2$. So far, uniqueness results are known for $k<1$, while existence holds for any $k \geqslant 1$ and $f$ positive in open sets compactly embedded in a neighbourhood of the boundary. We extend the uniqueness results to the $k \geqslant 1$ case and show, with an example, that existence does not hold if $f$ is zero near the boundary. We even deal with the uniqueness result when $f$ is replaced by a nonlinear term $\lambda u^{q}$ with $0<q<1$ and $\lambda>0$.

Keywords: nonlinear elliptic equations; singular natural growth gradient terms; comparison principle

2010 Mathematics subject classification: Primary 35J65; 35J75

Secondary 35A02

\section{Introduction}

Let $\Omega$ be a smooth bounded domain of $\mathbb{R}^{N}$ and $0 \lessgtr f \in L^{q}(\Omega)$, with $q>\frac{1}{2} N$, $N \geqslant 2$. We consider the boundary-value problem

$$
\left.\begin{array}{rlrl}
-\Delta u+g(u)|D u|^{2} & =f(x) & & \text { in } \Omega, \\
u & =0 & & \text { on } \partial \Omega,
\end{array}\right\}
$$

where the function $g:(0,+\infty) \rightarrow[0,+\infty)$ is unbounded at zero. Actually, this kind of singular elliptic equations with gradient terms is a classical problem. For the

*Present address: Dipartimento di Scienze di Base e Applicate per l'Ingegneria 'Sapienza', Università di Roma I, Via Antonio Scarpa 10, 00161 Roma, Italy (tommaso.leonori@sbai.uniroma1 .it).

(c) 2018 The Royal Society of Edinburgh 
existence of a solution $0<u \in H_{0}^{1}(\Omega) \cap L^{\infty}(\Omega)$ two thresholds appear naturally: the first one relies on an integrability condition on $\exp \left(-\int_{1}^{s} g(t) \mathrm{d} t\right)$ as $s \sim 0$, i.e. whether or not

$$
\exp \left(-\int_{1}^{s} g(t) \mathrm{d} t\right) \in L^{1}(0,1)
$$

is satisfied. Under this first threshold, a solution $u \in H_{0}^{1}(\Omega)$ for (1.1) exists even in a more general context (say, with a nonlinear differential operator instead of the Laplacian) and with a non-negative non-trivial datum $f \in L^{2 N /(N+2)}(\Omega)$ on the right-hand side (see, for example, [4]).

To prove the existence of a solution relaxing the growth condition on $g$ at 0 and overcoming the first threshold, i.e. when condition (1.2) is not satisfied, the datum $f(x)$ cannot degenerate to 0 inside $\Omega$. More precisely, $f$ has to satisfy

$$
f(x) \geqslant c_{\omega}>0, \quad \forall \omega \subset \subset \Omega .
$$

Then, a second natural threshold arises:

$$
\int_{0}^{1} \sqrt{g(s)} \mathrm{d} s<+\infty
$$

In [2] it is proved that the existence of a solution holds if (1.3) and (1.4) are satisfied. Otherwise (see $[2,9]$ ) non-existence of $H_{0}^{1}(\Omega)$ solutions occurs. In fact, as shown in [5], condition (1.3) is only needed in a neighbourhood of the boundary, i.e. it can be replaced by

$$
f(x) \geqslant c_{\omega}>0 \text { for any } \omega \subset \Omega_{\delta},
$$

where $\Omega_{\delta}=\{x \in \Omega: \operatorname{dist}(x, \partial \Omega)<\delta\}$ for some $\delta>0$.

In [6] lower-order terms of the form $g(u)|D u| \chi_{\{u>0\}}$ were considered, and solutions that can be zero on a closed 'large' set were obtained, even for $g$ negative. Solutions that change sign in the interior of $\Omega$ if $g$ is negative and $f$ changes sign were also obtained in [7].

As far as the uniqueness of solutions for (1.1) is concerned, the only known results about singular gradient terms that cover the case in which (1.2) is satisfied rely on $[1,3]$. To the best of our knowledge, it is unknown whether it is possible to go beyond the first threshold by proving the uniqueness of the solution.

The aim of this paper is to give a method of comparing a subsolution and a supersolution for (1.1) when we are beyond the threshold (1.2). The main difficulty with such a problem is the lack of a Hopf lemma due to the presence of the singular gradient term. Here we prove (via a suitable comparison with sub- and supersolutions) that solutions behave at the boundary as a suitable power of the distance to the boundary. This forces us to give a more accurate estimate of solutions near $\partial \Omega$, which removes the singularity of the lower-order term.

The paper is organized as follows. Section 2 is devoted to proving a uniqueness result for smooth solutions of (1.1) in the case where condition (1.2) is not satisfied. We also assume (1.5), which, as mentioned above, is needed to prove existence results if (1.2) fails. In $\S 3$, we prove with a counterexample that this condition is in some sense necessary for the existence. 


\section{Comparison principle and uniqueness}

In this section we prove a comparison principle for problem (1.1) in the case in which $g:(0,+\infty) \rightarrow[0,+\infty)$ is a continuous function, singular at 0 , such that $g(s) \simeq k / s, k \geqslant 1$. More precisely, we assume that the function $g$ satisfies the following hypothesis:

$$
\exists a_{0}>0 \text { and } \kappa \geqslant 1: \max \{\kappa-1,1\} \leqslant g(s) s \leqslant \kappa, \quad s \in\left(0, a_{0}\right) .
$$

Observe that, without loss of generality, we may assume that $\kappa>1$. Moreover, the above hypothesis implies that

$$
\mathrm{e}^{-G(s)} \notin L^{1}\left(0, a_{0}\right), \quad \text { where } G(s)=\int_{a_{0}}^{s} g(t) \mathrm{d} t .
$$

As far as the right-hand side of (1.1) is concerned, we assume that $f: \Omega \rightarrow[0,+\infty)$ verifies $f \in L_{\mathrm{loc}}^{1}(\Omega)$ and

$$
\exists \delta>0, \exists 0<\alpha \leqslant \beta, r \geqslant 0: \alpha \varphi_{1}^{r}(x) \leqslant f(x) \leqslant \beta \varphi_{1}^{r}(x) \text { in } \Omega_{\delta},
$$

where $\varphi_{1}$ is a positive eigenfunction corresponding to the first eigenvalue associated with $-\Delta$ in $\Omega$ with homogeneous Dirichlet boundary conditions. We point out that (2.2) implies, at least in the neighbourhood of the boundary of the form

$$
\Omega_{\delta}=\{x \in \Omega: \operatorname{dist}(x, \partial \Omega)<\delta\},
$$

for some $\delta>0$, the function $f$ satisfies (1.5).

In the following, given $\Omega$, a bounded open set of $\mathbb{R}^{N}$ with $N \geqslant 2$, we denote by $H_{\mathrm{c}}^{1}(\Omega)$ the space of functions that belong to $H_{0}^{1}(\Omega)$ with compact support in $\Omega$. First, we recall the meaning that we give to a subsolution and a supersolution of the singular equation

$$
-\Delta u+g(u)|D u|^{2}=f(x) \text { in } \Omega .
$$

Definition 2.1. Assume that $f \in L_{\text {loc }}^{1}(\Omega)$. Then, we say that

(i) $u \in H^{1}(\Omega) \cap C^{0}(\bar{\Omega})$ is a subsolution to $(2.3)$ if $u>0$ and

$$
\int_{\Omega} D u \cdot D \phi+\int_{\Omega} g(u)|D u|^{2} \phi \leqslant \int_{\Omega} f(x) \phi, \quad \forall \phi \in H_{c}^{1}(\Omega) \cap L^{\infty}(\Omega), \phi \geqslant 0,
$$

(ii) $v \in H^{1}(\Omega) \cap C^{0}(\bar{\Omega})$ is a supersolution to $(2.3)$ if $v>0$ and

$$
\int_{\Omega} D v \cdot D \phi+\int_{\Omega} g(v)|D v|^{2} \phi \geqslant \int_{\Omega} f(x) \phi, \quad \forall \phi \in H_{c}^{1}(\Omega) \cap L^{\infty}(\Omega), \phi \geqslant 0,
$$

(iii) $z \in H_{0}^{1}(\Omega) \cap C^{0}(\bar{\Omega})$ is a solution to (1.1) if it is both a subsolution and a supersolution to $(2.3)$.

REMARK 2.2. We stress that the definition of the solution (as well as those of the sub- and supersolution) requires that it belongs to $C^{0}(\bar{\Omega})$, which seems to be a 
restrictive assumption. As explained in [2, remark 2.6], because solutions of (1.1) are subsolutions of the Dirichlet problem associated with $-\Delta u=f$ in $\Omega$, following [8], we deduce that if $f \in L^{m}(\Omega)$ with $m>\frac{1}{2} N$, then $u$ is also continuous in $\bar{\Omega}$.

The main result we want to prove is the following.

THEOREM 2.3. Under the assumptions (2.1) and (2.2), there exists a unique positive $H_{0}^{1}(\Omega) \cap C^{0}(\bar{\Omega})$ solution to (1.1).

This is the direct consequence of a comparison result between super- and subsolutions to (2.3) with suitable boundary behaviour. In fact, our main effort will be to prove that any sub- or supersolution has the same behaviour at $\partial \Omega$.

Thus, we begin by defining some auxiliary functions and collecting together their main properties. We have already defined the function $G(s)=\int_{a_{0}}^{s} g(t) \mathrm{d} t$ for some $a_{0}>0$. Let us also consider the function $\psi(s)$ defined by

$$
\psi(s)=\int_{a_{0}}^{s} \mathrm{e}^{-G(t)} \mathrm{d} t, \quad s>0 .
$$

Observe that $\psi(s)$ is an increasing function and, assuming (2.1),

$$
\psi_{0} \equiv \lim _{s \rightarrow 0^{+}} \psi(s)=-\int_{0}^{a_{0}} \mathrm{e}^{-G(t)} \mathrm{d} t=-\infty
$$

and

$$
\psi_{\infty} \equiv \lim _{s \rightarrow+\infty} \psi(s)=\int_{a_{0}}^{+\infty} \mathrm{e}^{-G(t)} \mathrm{d} t>0,
$$

with $\psi_{\infty}$ possibly being infinite.

We next define a third function, with two parameters: for any $a, b>0$ we define $\varphi_{a, b}(s)$ (or $\varphi$ for brevity) as the solution of the following Cauchy problem:

$$
\left.\begin{array}{c}
\varphi^{\prime \prime}(s)=\left[g(\varphi(s)) \varphi^{\prime}(s)-g(s)\right] \varphi^{\prime}(s), \quad s \in\left(0, a_{0}\right), \\
\varphi\left(a_{0}\right)=a, \quad \varphi^{\prime}\left(a_{0}\right)=b .
\end{array}\right\}
$$

Observe that $\varphi$ solves

$$
\varphi^{\prime}(s)=b \mathrm{e}^{G(\varphi(s))-G(s)-G(a)} \quad \text { in }\left(0, a_{0}\right) .
$$

Thus, we have that

$$
\varphi^{\prime}(s) \mathrm{e}^{-G(\varphi(s))}=b \mathrm{e}^{-G(s)-G(a)} \quad \text { in }\left(0, a_{0}\right),
$$

and so, integrating the above identity between $a_{0}$ and $s$, we deduce that

$$
\psi(\varphi(s))-\psi\left(\varphi\left(a_{0}\right)\right)=\int_{\varphi\left(a_{0}\right)}^{\varphi(s)} \mathrm{e}^{-G(t)} \mathrm{d} t=b \mathrm{e}^{-G(a)} \int_{a_{0}}^{s} \mathrm{e}^{-G(t)} \mathrm{d} t=b \mathrm{e}^{-G(a)} \psi(s),
$$

i.e.

$$
\psi(\varphi(s))=\psi(a)+b \mathrm{e}^{-G(a)} \psi(s) .
$$

In the following lemma we list some useful properties of function $\varphi$ when condition (2.1) is satisfied. 
A uniqueness result for a singular elliptic equation with gradient term 987

LEMma 2.4. Under the hypothesis (2.1), the function $\varphi$ defined in (2.7) satisfies the following:

(i) $\varphi(s)$ is well defined for every $s \in\left(0, a_{0}\right)$;

(ii) $\lim _{s \rightarrow 0^{+}} \varphi(s)=0$;

(iii) $\varphi \in C^{2}\left(0, a_{0}\right)$ and $\varphi^{\prime \prime}(s)=\left(g(\varphi(s)) \varphi^{\prime}(s)-g(s)\right) \varphi^{\prime}(s)$ for every $s \in\left(0, a_{0}\right)$;

(iv) if $a<a_{0}$ and $b \mathrm{e}^{-G(a)} \geqslant 1$, then $0<\varphi(s) \leqslant s$ for every $s \in\left(0, a_{0}\right)$ and $\varphi^{\prime}$ is positive and bounded;

(v) if be $\mathrm{e}^{-G(a)} \geqslant(1+\varepsilon)^{\kappa-1}$ and $a \leqslant a_{0} /(1+\varepsilon)$ for some $\varepsilon>0$, then $0<(1+$ $\varepsilon) \varphi(s) \leqslant s$ for every $s \in\left(0, a_{0}\right)$;

(vi) if $b \mathrm{e}^{-G(a)}=(1+\varepsilon)^{\kappa-1}$ and $a=a_{0} /(1+\varepsilon)$, then $\varphi^{\prime}(s) \leqslant 1$ for every $s \in$ $\left(0, a_{0}\right)$.

Proof. Items (i)-(iii) are straightforward. In order to prove item (iv) we observe that

$$
\psi(a)+b \mathrm{e}^{-G(a)} \psi(s) \leqslant \psi(s), \quad s \in\left(0, a_{0}\right) .
$$

Thus, since $\psi^{-1}$ is increasing, we deduce that $\varphi(s) \leqslant s$. Taking into account that $\varphi^{\prime}(s)=b \mathrm{e}^{-G(a)} \mathrm{e}^{G(\varphi(s))-G(s)}$, we deduce also that $\varphi^{\prime}$ is positive and bounded.

Now we prove item (v). This is deduced from the inequality

$$
\psi(a)+b \mathrm{e}^{-G(a)} \psi(s) \leqslant \psi\left(\frac{1}{1+\varepsilon} s\right), \quad s \in\left(0, a_{0}\right) .
$$

Indeed, the function

$$
h(s)=\psi(a)+b \mathrm{e}^{-G(a)} \psi(s)-\psi\left(\frac{1}{1+\varepsilon} s\right)
$$

is increasing since

$$
\mathrm{e}^{G(s)} h^{\prime}(s)=b \mathrm{e}^{-G(a)}-\frac{1}{1+\varepsilon} \exp \left(G(s)-G\left(\frac{s}{1+\varepsilon}\right)\right) .
$$

Observe that if $g(s) s \leqslant \kappa$, then

$$
G(s)-G\left(\frac{s}{1+\varepsilon}\right)=\int_{s /(1+\varepsilon)}^{s} g(t) \mathrm{d} t \leqslant \ln (1+\varepsilon)^{\kappa} .
$$

In particular,

$$
\mathrm{e}^{G(s)} h^{\prime}(s) \geqslant b \mathrm{e}^{-G(a)}-(1+\varepsilon)^{\kappa-1} \geqslant 0 .
$$

Thus,

$$
h(s) \leqslant h\left(a_{0}\right)=\psi(a)-\psi\left(\frac{a_{0}}{1+\varepsilon}\right) \leqslant 0, \quad s \in\left(0, a_{0}\right) .
$$


Finally, item (vi) is deduced since $g(s) s \geqslant \kappa-1$ implies that

$$
\begin{aligned}
\varphi^{\prime}(s) & =(1+\varepsilon)^{\kappa-1} \mathrm{e}^{G(\varphi(s))-G(s)} \\
& \leqslant(1+\varepsilon)^{\kappa-1} \exp \left(G\left(\frac{s}{1+\varepsilon}\right)-G(s)\right) \\
& \leqslant(1+\varepsilon)^{\kappa-1} \mathrm{e}^{-(\kappa-1) \ln (1+\varepsilon)} \\
& =1 .
\end{aligned}
$$

REMARK 2.5. Let us explicitly compute the functions introduced in (2.6) and (2.7) when $g(s)=k / s$, with $k \geqslant 1$ : our model case (see $(*))$. We have that

$$
\psi(s)=\frac{a_{0}^{k}}{k-1}\left(a_{0}^{1-k}-s^{1-k}\right)
$$

and

$$
\varphi(s)=\left(b\left(\frac{a_{0}}{a}\right)^{k}\left(s^{1-k}-a_{0}^{1-k}\right)+a^{1-k}\right)^{-1 /(k-1)}
$$

if $k>1$, while

$$
\psi(s)=a_{0} \log \left(\frac{s}{a_{0}}\right) \quad \text { and } \quad \varphi(s)=a\left(\frac{s}{a_{0}}\right)^{b a_{0} / a}
$$

if $k=1$.

Now we are ready to prove the following comparison result between sub- and supersolutions of (2.3) that have a comparable behaviour on $\partial \Omega$. We define $d(x)=$ $\operatorname{dist}(x, \partial \Omega)$.

THEOREM 2.6. Let $u$ and $v$ respectively be a subsolution and a supersolution of (2.3) with $g$ satisfying (2.1) and $f \in L^{1}(\Omega), f \geqslant 0$. Suppose that

$$
\liminf _{d(x) \rightarrow 0} \frac{v(x)}{u(x)} \geqslant 1 .
$$

Then $u \leqslant v$ in $\Omega$.

REMARK 2.7. Although the main interest of the previous theorem, due to the singularity, is when $u=v=0$ on $\partial \Omega$, we observe that the above result does not depend on the fact that $u$ and $v$ vanish on $\partial \Omega$.

Proof. First, we fix $a_{0}>\|u\|_{L^{\infty}(\Omega)}$ and for any $\varepsilon>0$ we denote by $\varphi_{\varepsilon}$ the function $\varphi_{a, b}$ with $a=a_{0} /(1+\varepsilon)$ and $b \mathrm{e}^{-G(a)}=(1+\varepsilon)^{\kappa-1}$. Recall that, under assumption (2.1) we have that

$$
\varphi_{\varepsilon}\left(a_{0}\right)=\frac{a_{0}}{1+\varepsilon}, \quad \varphi_{\varepsilon}^{\prime}\left(a_{0}\right) \mathrm{e}^{-G\left(\varphi_{\varepsilon}\left(a_{0}\right)\right)}=(1+\varepsilon)^{\kappa-1}
$$

and

$$
\varphi_{\varepsilon}(s)=\psi^{-1}\left(\psi\left(\frac{a_{0}}{1+\varepsilon}\right)+(1+\varepsilon)^{\kappa-1} \psi(s)\right) .
$$


We claim that the function $u_{\varepsilon}=\varphi_{\varepsilon}(u)$ is still a subsolution of (2.3) satisfying

$$
\liminf _{d(x) \rightarrow 0} \frac{u}{u_{\varepsilon}} \geqslant(1+\varepsilon) .
$$

Indeed, using lemma 2.4, we have that $u_{\varepsilon}=\varphi_{\varepsilon}(u) \in H^{1}(\Omega) \cap C^{0}(\bar{\Omega}), u_{\varepsilon}>0$. This verifies

$$
\begin{aligned}
-\Delta u_{\varepsilon}+ & g\left(u_{\varepsilon}\right)\left|D u_{\varepsilon}\right|^{2}-f(x) \\
& \leqslant-f(x)\left[1-\varphi_{\varepsilon}^{\prime}(u)\right]+|D u|^{2}\left[-g(u) \varphi_{\varepsilon}^{\prime}(u)-\varphi_{\varepsilon}^{\prime \prime}(u)+g\left(\varphi_{\varepsilon}(u)\right) \varphi_{\varepsilon}^{\prime 2}(u)\right] \\
& =-f(x)\left[1-\varphi_{\varepsilon}^{\prime}(u)\right] .
\end{aligned}
$$

Using the fact that $\varphi_{\varepsilon}^{\prime}(u) \leqslant 1$ we deduce that the right-hand side in $(2.12)$ is nonpositive, and consequently $u_{\varepsilon}$ is a subsolution.

The second part of the statement is deduced from lemma 2.4(v). Indeed, since $(1+\varepsilon) \varphi_{\varepsilon}(u) \leqslant u$, we have

$$
\liminf _{d(x) \rightarrow 0} \frac{u}{\varphi_{\varepsilon}(u)} \geqslant(1+\varepsilon)
$$

and the claim is proved.

Combining (2.9) with (2.11) we deduce that

$$
\liminf _{d \rightarrow 0} \frac{v}{u_{\varepsilon}}=\liminf _{d \rightarrow 0} \frac{v}{u} \frac{u}{u_{\varepsilon}} \geqslant(1+\varepsilon) \liminf _{d \rightarrow 0} \frac{v}{u}>1,
$$

and consequently there exists $\Omega_{\varepsilon} \subset \Omega$ such that $u_{\varepsilon}-v \leqslant 0$ in $\Omega_{\varepsilon}$.

Thus, with $\psi$ defined in $(2.6)$, we have that $\left[\psi\left(u_{\varepsilon}\right)-\psi(v)\right]^{+}$is supported in $\Omega \backslash$ $\Omega_{\varepsilon}$. This implies that the function $\phi=\mathrm{e}^{-G\left(u_{\varepsilon}\right)}\left[\psi\left(u_{\varepsilon}\right)-\psi(v)\right]^{+}$belongs to $H_{\mathrm{c}}^{1}(\Omega) \cap$ $L^{\infty}(\Omega)$ and it can be taken as test function in the formulation of $u_{\varepsilon}$, so that

$$
\int_{\Omega} D \psi\left(u_{\varepsilon}\right) \cdot D\left[\psi\left(u_{\varepsilon}\right)-\psi(v)\right]^{+} \leqslant \int_{\Omega} f(x) \mathrm{e}^{-G\left(u_{\varepsilon}\right)}\left[\psi\left(u_{\varepsilon}\right)-\psi(v)\right]^{+} .
$$

Analogously, for the supersolution, we take $\mathrm{e}^{-G(v)}\left[\psi\left(u_{\varepsilon}\right)-\psi(v)\right]^{+}$as test function, and we have that

$$
\int_{\Omega} D \psi(v) \cdot D\left[\psi\left(u_{\varepsilon}\right)-\psi(v)\right]^{+} \geqslant \int_{\Omega} f(x) \mathrm{e}^{-G(v)}\left[\psi\left(u_{\varepsilon}\right)-\psi(v)\right]^{+} .
$$

Thus, subtracting the two inequalities, we deduce that

$$
\int_{\Omega}\left|D\left[\psi\left(u_{\varepsilon}\right)-\psi(v)\right]^{+}\right|^{2} \leqslant \int_{\Omega} f(x)\left[\psi^{\prime}\left(u_{\varepsilon}\right)-\psi^{\prime}(v)\right]\left[\psi\left(u_{\varepsilon}\right)-\psi(v)\right]^{+} \leqslant 0,
$$

and consequently $u_{\varepsilon} \leqslant v$ also in $\Omega \backslash \Omega_{\varepsilon}$ for all $\varepsilon>0$. Letting $\varepsilon$ vanish, we have that $u \leqslant v$ in $\Omega$.

REMARK 2.8. Let us stress that we are left with the case $k=1$ in $(*)$. In this case it is not so hard to prove that $\varphi_{\varepsilon}(s)$ is replaced by $s^{1+\varepsilon}$, i.e. if $u$ is a subsolution, then $u_{\varepsilon}=u^{1+\varepsilon}$ is still a subsolution. 
Indeed, assume that $\|u\|_{L^{\infty}(\Omega)} \leqslant a<1$ (otherwise we can reduce to this case after a rescaling of the equation), and define $u_{\varepsilon}=u^{1+\varepsilon}$ for $\varepsilon>0$. Hence,

$$
-\Delta u_{\varepsilon}+\frac{1}{u_{\varepsilon}}\left|D u_{\varepsilon}\right|^{2}-f(x) \leqslant-f(x)\left[1-(1+\varepsilon) u^{\varepsilon}\right] \leqslant 0,
$$

i.e. $u_{\varepsilon}$ is a subsolution. Moreover, if $u=0$ on $\partial \Omega$, we observe that

$$
\liminf _{d(x) \rightarrow 0} \frac{u}{u_{\varepsilon}}=+\infty .
$$

The next step in order to prove the uniqueness of the solution of (1.1) is to prove that if $f$ satisfies (2.2), all the solutions have a boundary behaviour comparable with $\varphi_{1}^{2+r}$, where, as mentioned in $\S 1, \varphi_{1}$ denotes a positive eigenfunction corresponding to the first eigenvalue associated with $-\Delta$ in $\Omega$ with homogeneous Dirichlet boundary conditions. We recall the following result concerning $\varphi_{1}$.

Lemma 2.9. Let $\gamma>0$. Then there exists $0<\gamma_{1} \leqslant \gamma_{2}<+\infty$ such that

$$
\gamma_{1} \leqslant \varphi_{1}^{2}+\gamma\left|D \varphi_{1}\right|^{2} \leqslant \gamma_{2} \quad \text { in } \Omega .
$$

Proof. The second inequality is trivial, since we can choose

$$
\gamma_{2}=\left\|\varphi_{1}\right\|_{L^{\infty}(\Omega)}^{2}+\gamma\left\|D \varphi_{1}\right\|_{L^{\infty}(\Omega)}^{2} .
$$

For the other one, we argue by contradiction. Assume that $\inf _{\Omega} \varphi_{1}^{2}+\gamma\left|D \varphi_{1}\right|^{2}=0$. As $\varphi_{1}^{2}+\gamma\left|D \varphi_{1}\right|^{2}$ is smooth in $\bar{\Omega}$, its minimum is equal to zero. In addition, the minimum point cannot lie in the interior of $\Omega$, since $\varphi_{1}>0$ there. On the other hand, on $\partial \Omega$ we have (via the Hopf lemma) that $\partial \varphi_{1} /\left.\partial \nu\right|_{\partial \Omega}<0$, and consequently $\left|D \varphi_{1}\right|^{2}>0$ on $\partial \Omega$.

Let us now state our boundary behaviour result.

THEOREM 2.10. Let $u$ be any solution to (1.1) with $g$ and $f$ satisfying (2.1) and (2.2), respectively. Then there exist $M, \eta>0$ depending only on $\Omega$ and $r \geqslant 0$, such that

$$
\eta \leqslant \frac{u}{\varphi_{1}^{r+2}} \leqslant M \quad \text { in } \Omega_{\delta}
$$

Proof. The idea is to exploit the comparison principle in theorem 2.6.

First, consider the following supersolution of (2.3) in $\Omega_{\delta}: \bar{v}_{\varepsilon}=M\left(\varepsilon+\varphi_{1}\right)^{r+2}$ for some $M>0$ and $\varepsilon>0$. Using the fact that $f$ satisfies (2.2), direct computations show that it turns out to be a supersolution when $M$ is chosen sufficiently large, uniformly with respect to $\varepsilon$. Moreover, since, for any $\varepsilon>0, \bar{v}_{\varepsilon}=M \varepsilon^{r+2}$ on $\partial \Omega$, we have, for any solution $u$ of (1.1),

$$
\liminf _{d(x) \rightarrow 0} \frac{\bar{v}_{\varepsilon}}{u}=+\infty, \quad \liminf _{d(x) \rightarrow \delta} \frac{\bar{v}_{\varepsilon}}{u} \geqslant M \max _{d(x)=\delta} \frac{\left(\varphi_{1}(x)\right)^{r+2}}{u(x)}>1 .
$$

Thus, thanks to theorem 2.6, $\bar{v}_{\varepsilon} \geqslant u$ in $\Omega_{\delta}$. Letting $\varepsilon$ go to 0 , we deduce that $u \leqslant M \varphi_{1}^{r+2}$ in $\Omega_{\delta}$. 
Now, we consider $\Omega_{\delta}^{\rho}=\{x \in \Omega: \rho<d(x)<\delta\}$ for any $0<\rho<\delta$, and observe that $u$ is a supersolution of the problem

$$
\left.\begin{array}{rlrl}
-\Delta z+g(z)|D z|^{2} & =f(x) & & \text { in } \Omega_{\delta}^{\rho}, \\
z & =0 & & \text { on } d(x)=\rho, \\
z & =u & & \text { on } d(x)=\delta,
\end{array}\right\}
$$

We denote by $\varphi_{1, \rho}$ the first positive eigenfunction, with $\left\|\varphi_{1, \rho}\right\|_{L^{\infty}(\Omega)}=1$, associated with $-\Delta$ in $\Omega^{\rho}=\{x \in \Omega: \rho<d(x)\}$ with zero Dirichlet boundary conditions, extended to 0 in $\Omega \backslash \Omega^{\rho}$, and denote by $\lambda_{1, \rho}$ its corresponding eigenvalue. Observe that $\varphi_{1, \rho} \rightarrow \varphi_{1}$ in $C^{0}(\bar{\Omega}) \cap C_{\mathrm{loc}}^{1}(\Omega)$, due to the linearity of the problem and since both $\varphi_{1}$ and $\varphi_{1, \rho}$ are normalized in $L^{\infty}$ to 1 . Moreover, $\lambda_{1, \rho} \rightarrow \lambda_{1}$ as $\rho \rightarrow 0$.

We can choose $\eta>0$ independent on $\rho$ such that the function $\underline{u}_{\rho}=\eta \varphi_{1, \rho}^{r+2}$ is a subsolution of (2.17) for every $\rho \in\left[0, \delta_{0}\right]$ for some $\delta_{0} \in(\rho, \delta)$. Indeed, note that

$-\Delta \underline{u}_{\rho}+g\left(\underline{u}_{\rho}\right)\left|D \underline{u}_{\rho}\right|^{2}-f(x) \leqslant 2 \eta \lambda_{1, \rho} \varphi_{1, \rho}^{q}\left[\varphi_{1, \rho}^{2}+\frac{2 \kappa-1}{\lambda_{1, \rho}}\left|D \varphi_{1, \rho}\right|^{2}\right]-\alpha \varphi_{1, \rho}^{r} \quad$ in $\Omega_{\delta}^{\rho}$, so that $\underline{u}_{\rho}$ is a subsolution of (2.17) if

$$
0<\eta \leqslant \min \left\{\frac{\alpha}{2 \lambda_{1, \rho} c_{1}}, \inf _{d(x)=\delta} u(x)\right\}
$$

where

$$
c_{1}=\max _{\rho \in[0, \delta]} \max _{x \in \overline{\Omega^{\rho}}}\left[\varphi_{1, \rho}^{2}+\frac{2 \kappa-1}{\lambda_{1, \rho}}\left|D \varphi_{1, \rho}\right|^{2}\right]
$$

(see lemma 2.9).

Observe that $0<c_{1}<\infty$, since $\varphi_{1, \rho} \rightarrow \varphi_{1}$ in $C^{0}(\bar{\Omega}) \cap C_{\mathrm{loc}}^{1}(\Omega)$.

Now, applying theorem 2.6, we deduce that $\underline{u}_{\rho} \leqslant u$ in $\Omega_{\delta}^{\rho}$, as in this case $\liminf _{d_{\rho} \rightarrow 0}\left(u / \underline{u}_{\rho}\right)=+\infty$.

Thus, letting $\rho$ vanish, we obtain

$$
\eta \varphi_{1}^{r+2}=\underline{u}_{0}=\lim _{\rho \rightarrow 0} \eta \varphi_{1, \rho}^{r+2} \leqslant u \quad \text { in } \Omega_{\delta} .
$$

Now we are ready to prove a uniqueness result for solutions of (1.1).

Proof of theorem 2.3. Assume problem (1.1) has two solutions, $u$ and $v$. According to theorem 2.10 ,

$$
0<\frac{\eta}{M} \leqslant \frac{u}{v} \leqslant \frac{M}{\eta} \quad \text { in } \Omega_{\delta}
$$

Fix any $\varepsilon>0$, and consider the function

$$
\varphi_{\varepsilon}(s)=\psi^{-1}\left(\psi\left(\frac{a_{0}}{1+\varepsilon}\right)+(1+\varepsilon)^{\kappa-1} \psi(s)\right)
$$

for some $a_{0}>\|u\|_{L^{\infty}(\Omega)},\|v\|_{L^{\infty}(\Omega)}$ and with $\psi$ as in (2.6). Also, define, for any $m \in \mathbb{N}$,

$$
u_{\varepsilon}=\varphi_{\varepsilon}^{[m]}(u), \quad \text { where } \varphi_{\varepsilon}^{[m]}(s)=\underbrace{\varphi_{\varepsilon} \circ \varphi_{\varepsilon} \circ \cdots \circ \varphi_{\varepsilon}}_{m \text { times }}(u) .
$$


First, observe that, given any subsolution $u \in H_{0}^{1}(\Omega) \cap C^{0}(\bar{\Omega})$ of (1.1), by induction any $\varphi_{\varepsilon}^{[m]}(u)$, with $m \in \mathbb{N}$ is still an $H_{0}^{1}(\Omega) \cap C^{0}(\bar{\Omega})$ subsolution. Moreover, $\varphi_{\varepsilon}^{[m]}(u)$ also satisfies

$$
\liminf _{d(x) \rightarrow 0^{+}} \frac{v}{u_{\varepsilon}} \geqslant(1+\varepsilon)^{m} \liminf _{d(x) \rightarrow 0^{+}} \frac{v}{u} \geqslant \frac{\eta}{M}(1+\varepsilon)^{m} ;
$$

note that $m$ can be chosen (depending only on $\eta$ and $M$ ) such that the right-hand side above is strictly bigger than 1 . We can now apply theorem 2.6 and we have that $u_{\varepsilon} \leqslant v$; letting $\varepsilon$ vanish, we deduce that $u \leqslant v$. By exchanging the roles of $u$ and $v$, we get the reverse inequality.

In particular, we have the following corollary.

Corollary 2.11. There exists a unique positive $H^{1}(\Omega) \cap C^{0}(\bar{\Omega})$ solution to

$$
\begin{aligned}
-\Delta u+\frac{k}{u}|D u|^{2} & =f & & \text { in } \Omega, \\
u & =0 & & \text { on } \partial \Omega,
\end{aligned}
$$

with $k \geqslant 1$ and $f$ as in (2.2).

With the same technique we can prove uniqueness if we replace the right-hand side by a power nonlinearity. More specifically, we want to prove the following result.

Theorem 2.12. Assume that $u, v \in H_{0}^{1}(\Omega) \cap C^{0}(\bar{\Omega})$ are two solutions of

$$
\left.\begin{array}{rlrl}
-\Delta z+g(z)|D z|^{2} & =a(x) z^{q} & & \text { in } \Omega, \\
z & =0 & & \text { on } \partial \Omega,
\end{array}\right\}
$$

with $q \in(0,1)$ and $0<a_{1} \leqslant a(x) \leqslant a_{2}$ and $g$ as in (2.1). Then $u \equiv v$ in $\Omega$.

Proof. We reproduce the proofs of theorems 2.3 and 2.10 .

We first want to argue as in theorem 2.6 in order to deduce that any pair of sub- and supersolutions (in the sense of definition 2.1 with $f(x)$ replaced by $a(x) u^{q}$ in (2.4) and by $a(x) v^{q}$ in (2.5)), which are comparable on $\partial \Omega$ (i.e. such that (2.9) holds), are ordered in $\Omega$. Hence, it is not difficult to see that, given any subsolution $u$, using lemma $2.4(\mathrm{iv})-(\mathrm{vi}), \varphi_{\varepsilon}(u)$ is still a subsolution for every $\varepsilon>0$, where (2.13) holds. Hence, we deduce the comparison near $\partial \Omega$.

Moreover, once again by lemma 2.4(vi), we can argue as in (2.14) and (2.15) that the comparison still holds in the interior.

Thus, given any sub- and supersolutions, we want to prove that necessarily (2.9) holds. To do this, we just find a suitable couple of sub- and supersolutions that behave in a comparable way on $\partial \Omega$. Observe that, using the regularity properties of $\varphi_{1}$, one can prove that

$$
\exists 0<\alpha \leqslant \beta: 0<\alpha \leqslant \frac{2 \lambda_{1}}{1-q} \varphi_{1}^{2}+\frac{2}{1-q}\left|D \varphi_{1}\right|^{2} \leqslant \beta .
$$

We set $\bar{u}=M\left(\varepsilon+\varphi_{1}\right)^{2 /(1-q)}$ with $M>0$ (to be chosen) and $\varepsilon>0$, so that

$$
-\Delta \bar{u}+\frac{1}{\bar{u}}|D \bar{u}|^{2}-a(x) \bar{u}^{q} \geqslant M \varphi_{1}^{2 q /(1-q)}\left[\frac{2 \lambda_{1}}{1-q} \varphi_{1}^{2}+\frac{2}{1-q}\left|D \varphi_{1}\right|^{2}-a_{2} M^{q-1}\right] \quad \text { in } \Omega \text {. }
$$


Since $q-1<0$, it is sufficient to choose $M \geqslant\left(\alpha / a_{2}\right)^{1 /(q-1)}$ and $\bar{u}$ turns out to be a supersolution to (2.18). Moreover, $\bar{u}=M \varepsilon^{2 /(1-q)}>0$ on $\partial \Omega$.

Arguing as in theorem 2.10, we can also construct, in a neighbourhood of the boundary, a subsolution that behaves like $\underline{u}=\eta \varphi_{1}^{2 /(1-q)}$, with $\eta>0$ and $\eta \leqslant$ $\left(\alpha / a_{1}\right)^{1 /(q-1)}$.

We can now repeat the same proof for theorem 2.3 to prove uniqueness.

\section{A counterexample}

In order to obtain the existence of the solution, we prove here that condition (1.5) cannot be relaxed, at least in dimension 1 , if $\mathrm{e}^{-G(s)}$ is not integrable at zero. This is a consequence of the following result.

TheOREM 3.1. Assume that $f \in C(0,1), f(x) \geqslant 0, f \not \equiv 0$ and $f(x) \equiv 0$ if $x \in(0, \varepsilon)$ for some $\varepsilon \in(0,1)$. There is no positive solution $u \in H_{0}^{1}(0,1)$ of the problem

$$
-u^{\prime \prime}(x)+\frac{k}{u(x)} u^{\prime 2}(x)=f(x), \quad x \in(0,1), \quad u(0)=u(1)=0 .
$$

Proof. We argue by contradiction, assuming that such a solution exists. Then

$$
-u^{\prime \prime}(x)+\frac{k}{u(x)} u^{\prime}(x)^{2}=0, \quad x \in(0, \varepsilon) \text { for some } \varepsilon>0 .
$$

We recall first that a solution has to be positive in $(0,1)$, and moreover that $H^{1}(0,1)$ is embedded in $A C(0,1)$. Consequently, we deduce that $u(x) \in W^{2,1}(0,1)$, which implies that $u^{\prime} \in A C$ and thus, by using the above equation once again, that $u \in C^{2}(0,1)$.

In particular, $u$ is a convex function in $(0, \varepsilon)$. Then $u^{\prime}$ is non-decreasing in this interval, which ensures the existence of $u^{\prime}(0)$. Moreover, $u^{\prime}(0) \geqslant 0$, since otherwise $u$ would be negative in a (right) neighbourhood of 0 (since $u(0)=0)$. If $u^{\prime}(t)=0$ for some $t \in(0, \varepsilon)$, then $u^{\prime}(x) \equiv 0$ for every $x \in(0, t)$, which implies that $u(x)=$ $u(0)=0$ for every $x \in(0, t)$, contradicting the condition that $u>0$. Thus, we may assume that $u^{\prime}(x)>0$ for every $x \in[0, \varepsilon)$.

Multiplying the equation by $1 / u^{\prime}(x)$ we have

$$
k(\ln u(x))^{\prime}=k \frac{u^{\prime}(x)}{u(x)}=\frac{u^{\prime \prime}(x)}{u^{\prime}(x)}=\left(\ln u^{\prime}(x)\right)^{\prime}, \quad x \in(0, \varepsilon),
$$

or, equivalently, for some positive constant $c>0, u^{\prime}(x) / u^{k}(x)=c$ for every $x \in$ $(0, \varepsilon)$.

If $k=1$, this implies that there exists a positive constant $A>0$ such that $u(x)=A \mathrm{e}^{c x}$ for every $x \in(0, \varepsilon)$. Thus, the claim follows since the condition $u(0)=0$ is violated.

Otherwise, if $k>1$, we integrate the differential identity $u^{\prime}(x) / u^{k}(x)=c$, and we deduce that

$$
\frac{1}{1-k} u^{1-k}(x)=c x+c_{1} \quad c>0, c_{1} \in \mathbb{R},
$$

which is not compatible with the initial condition $u(0)=0$. 


\section{Acknowledgements}

J.C. is partly supported by the Junta de Andalucía, Grant no. FQM-194 (Spain), and MINECO-FEDER Grant no. MTM2015-68210-P (Spain). T.L. is partly supported by the Junta de Andalucía, Grant no. FQM-116 (Spain), and MINECOFEDER Grant no. MTM2015-68210-P (Spain).

\section{References}

1 D. Arcoya and S. Segura de León. Uniqueness of solutions for some elliptic equations with a quadratic gradient term. ESAIM: Control Optim. Calc. Variations 2 (2010), 327-336.

2 D. Arcoya, J. Carmona, T. Leonori, P. J. Martínez-Aparicio, L. Orsina and F. Petitta. Existence and nonexistence of solutions for singular quadratic quasilinear equations. $J$. Diff. Eqns 246 (2009), 4006-4042.

3 D. Arcoya, J. Carmona and P. J. Martínez-Aparicio. Comparison principle for elliptic equations in divergence with singular lower order terms having natural growth. Commun. Contemp. Math. 19 (2017), 1650013.

4 L. Boccardo. Dirichlet problems with singular and quadratic gradient lower order terms. ESAIM: Control Optim. Calc. Variations 14 (2008), 411-426.

5 J. Carmona, P. J. Martínez-Aparicio and J. D. Rossi. A singular elliptic equation with natural growth in the gradient and a variable exponent. NoDEA. Nonlin. Diff. Eqns Applic. 22 (2015), 1935-1948.

6 D. Giachetti and F. Murat. An elliptic problem with a lower order term having singular behaviour. Boll. UMI B 2 (2009), 349-370.

7 D. Giachetti, F. Petitta and S. Segura de Leon. Elliptic equations having a singular quadratic gradient term and a changing sign datum. Commun. Pure Appl. Analysis 11 (2012), 1875-1895.

8 O. Ladyzenskaya and N. Ural'tseva. Linear and quasilinear elliptic equations (Engl. transl.) (New York: Academic Press, 1968).

9 W. Zhou, X. Wei and X. Qin. Nonexistence of solutions for singular elliptic equations with a quadratic gradient term. Nonlin. Analysis 75 (2012), 5845-5850. 